\title{
VON EINIGEN NERVENPUNKTEN \\ DES DENKENS AUF FREIHEIT \\ UND DEN LETZTEN SCHLUPFWINKELN \\ DES WIDERSTANDS. BEMERKUNGEN \\ ZUR PRAKTISCHEN PHILOSOPHIE \\ THEODOR W. ADORNOS
}

PIERRE BUHLMANN

\begin{abstract}
:
This paper is to examine how an Adornian moral philosophy could be conceived, in defiance of the ascertainment by Adorno himself that the realisation of such a practical philosophy has been made impossible today. At its heart is the idea of a reflexive subjectivity able to fully apprehend its object, and thus capable of ethical acts that are an expression of resistance through the execution of particular negation.
\end{abstract}

Pourl.

\section{Erster Teil. Negative Dialektik und der Zerfall des Systems}

1. Subjekt - Objekt

Grundlegend für das Verständnis adornitischen Denkens ist seine Auffassung des Subjekt-Objekt-Problems und ihrer Vermittlung. In der Tat durchdringt dieser Bereich alle von Adorno behandelten Themen, von der Erkenntnistheorie bis zur Moralphilosophie. Hierbei ist nicht nur bedeutsam, dass Adornos dialektischer Materialismus eine Prävalenz des Objekts über das Subjekt gegen den klassischen 
deutschen Idealismus ins Felde führt, sondern auch die Art, in der die Vermittlung der beiden ineinander gedacht wird. Deshalb soll dieses Problem im Folgenden vorgestellt werden.

In Negative Dialektik ${ }^{1}$ schreibt Adorno bezüglich der Vermittlung von Subjekt und Objekt:

Vermöge der Ungleichheit im Begriff der Vermittlung fällt das Subjekt ganz anders ins Objekt als dieses in jenes. Objekt kann nur durch Subjekt gedacht werden, erhält sich aber diesem gegenüber immer als Anderes; Subjekt jedoch ist der eigenen Beschaffenheit nach vorweg auch Objekt. Vom Subjekt ist Objekt nicht einmal als Idee wegzudenken; aber vom Objekt Subjekt. Zum Sinn von Subjektivität rechnet es, auch Objekt zu sein; nicht ebenso zum Sinn von Objektivität, Subjekt zu sein. (ND, 184)

Dass das Objekt dem Subjekt übergeordnet ist, zeigt der Umstand an, dass Objekt theoretisch ohne Subjekt gedacht werden kann, der umgekehrte Fall jedoch nicht möglich ist. Der Einschub theoretisch meint in diesem Zusammenhang, dass eine solche Hierarchisierung keine absolute, unabhängige Dualität der beiden Komplexe heraufbeschwören will (anders ausgedrückt, kann es in der Praxis kein Objekt ohne Vermittlung eines Subjekts geben), sondern lediglich ausdrücken möchte, dass jedes Subjekt, bevor es als Subjekt konstituiert ist, notwendig immer schon Objekt ist. Trotzdem beide also immer als vermittelt zusammengedacht werden müssen (es kann aus dialektischer Sicht somit keine Trennung geben), fallen sie dennoch wiederum nicht ineinander. Das meint der Umstand, dass das Objekt des Subjekts bedarf, durch das es gedacht wird, es sich diesem jedoch „immer als Anderes" darstellt. ${ }^{2}$

Hier nun kommt Adornos negative Dialektik ins Spiel, als das Denken, dass es vollbringen kann, die Vermittlung zwischen Subjekt und Objekt angemessen auszudrücken, sowie ebenfalls als das Denken, dass als einziges diese objektale Alterität berührt. Eine solche Dialektik vollbringt damit eine aufklärerische Geste gegen die Aufklärung, die sonst aufgrund der traditionellen begrifflichen Vormacht des Subjekts dialektisch in Mythos zurückschlägt. Dergestalt ist sie

\footnotetext{
Adorno, Theodor W.: Negative Dialektik, Suhrkamp, Frankfurt a. M. 2003 [1970]. Im Folgenden werden die Referenzen zu diesem Werk in Klammern im Text gegeben, versehen mit dem Kürzel „ND“, gefolgt von der Seitenzahl.

2 Hierzu auch folgendes Zitat Adornos: „Vermittlung des Objekts besagt, dass es nicht statisch, dogmatisch hypostasiert werden darf, sondern nur in seiner Verflechtung mit Subjektivität zu erkennen sei; Vermittlung des Subjekts, dass es ohne das Moment der Objektivität buchstäblich nicht wäre. Index für den Vorrang des Objekts ist die Ohnmacht des Geistes in all seinen Urteilen wie bis heute in der Einrichtung der Realität." (ND, 187)
} 
„Entmythologisierung nicht mehr nur als reductio ad hominem, sondern auch umgekehrt als reductio hominis, als Einsicht in den Trug des zum Absoluten sich stilisierenden Subjekts. Das Subjekt ist die späte und dennoch der ältesten gleiche Gestalt des Mythos" (ND, 187), wobei Mythos hier die ewige Rückkehr des Immergleichen im Identitätsdenken meint. Dem gegenüber

[ $m]$ uss [das Subjekt] am Nichtidentischen wiedergutmachen, was es daran verübt hat. Dadurch gerade wird es frei vom Schein seines absoluten Fürsichseins. Er seinerseits ist Produkt des identifizierenden Denkens, das je mehr es eine Sache zum bloßen Exempel seiner Art oder Gattung entwertet, desto mehr wähnt, es als solches ohne subjektiven Zusatz zu haben. (ND, 149)

Die Schwierigkeit resultiert hierbei daraus, dass das Nichtidentische nicht einfach mit dem Objekt gleichzusetzen ist. Vielmehr ist das Nichtidentische der überschüssige Rest, der bei der verallgemeinernden Begrifflichmachung immer und notgedrungen aus dem Begriff herausfällt; das, was mangels Respekt des Begriffs vor dem Einzelnen in diesem unterdrückt wird, was somit aber gleichzeitig dessen idiosynkratischstes Bestandteil darstellt. ${ }^{3}$ Gerade deshalb betont Adorno auch die Andersheit des Objekts, da dieses in Wahrheit ein Zusammenschießen aus Begriff und Nichtidentischem ist. Dies jedoch ist von der Philosophie, deren Definition nach Adorno angegeben werden könnte als dasjenige Denken, welches das Nichtbegriffliche mithilfe des Begriffs und durch den Begriff zu denken sich immerzu bemüht ${ }^{4}$, bislang nie ausreichend beachtet worden. Das ist auch der Grund, warum „[a]n der Zweiheit von Subjekt und Objekt [...] kritisch festzuhalten [ist $[\ldots]$ “ (ND, 177). Denn

beide Begriffe sind entsprungene Reflexionskategorien, Formeln für ein nicht zu Vereinendes; kein Positives, keine primären Sachverhalte, sondern negativ durchaus, Ausdruck einzig der Nichtidentität. Trotzdem ist die Differenz von Subjekt und Objekt auch nicht ihrerseits einfach zu negieren. Weder sind sie letzte Zweiheit, noch verbirgt hinter ihnen sich letzte Einheit. Sie konstituieren ebenso sich durch einander, wie sie vermöge solcher Konstitution auseinandertreten. (ND, 176)

\footnotetext{
Cf. ND, 164.
}

4 In diesem Zusammenhang führt Adorno in seiner Vorlesung zur Metaphysik aus: „Es scheint mir das Einzigartige an den philosophischen Begriffen zu sein [...], dass die Philosophie die sonderbare Eigenschaft hat, dass sie zwar selber verstrickt ist, dass sie zwar selber in dem Glashaus unserer Konstitution und unserer Sprache eingesperrt ist; dass sie aber trotzdem immer wieder vermag, über sich selbst hinaus, über diese Begrenzung hinaus und durch ihr Glashaus hindurch zu denken." Adorno: Metaphysik. Begriff und Probleme, Suhrkamp, Frankfurt a. M. 2006 [1965], S. 108. 


\section{Dialektik und Negativität}

Um die dargestellte Vermittlung so zu denken, dass dem Nichtidentischen auch in einem begrifflichen Denken Gerechtigkeit widerfährt, braucht es also negative Dialektik. Ihre Verfahrensweise beschreibt Adorno, wenn er ausführt:

[i]hre Bewegung tendiert nicht auf Identität in der Differenz jeglichen Gegenstandes von seinem Begriff; eher beargwöhnt sie Identisches. Ihre Logik ist eine des Zerfalls: der zugerüsteten und vergegenständlichten Gestalt der Begriffe, die zunächst das erkennende Subjekt unmittelbar sich gegenüber hat. Deren Identität mit dem Subjekt ist die Unwahrheit. Mit ihr schiebt sich die subjektive Präformation des Phänomens vor das Nichtidentische daran, vors individuum ineffabile. (ND, 148)

Eine solche Dialektik ist negativ, weil sie die Widersprüche, die sie vorfindet, nicht zwanghaft auflösen will, indem sie über sie hinausgeht, sondern sich auf ebendieses objektive kontradiktorische Moment konzentriert. Dies geschieht, um dem Nichtidentischen zu seinem Recht zu verhelfen, d.h. es nicht zu identifizieren, sondern es als Nichtidentisches zu enthüllen. In der Tat kann nur im Aufzeigen des Widersprüchlichen im Moment des Zerfalls (von Begriff und Sache) das Nichtidentische ebenso wie das Utopische sich in einem Aufblitzen des unsagbaren Überschusses zeigen. Denn da „, $[d]$ as Einzelne [...] mehr [ist $]$ sowohl wie weniger als seine allgemeine Bestimmung" (ND, 154), kann es nie einfach im Begriff aufgehen, d.h. naiv (in Sprache) sichtbar werden, sondern bedarf des negativ dialektischen Verfahrens.

Der Zerfall wiederum entsteht durch die Dynamisierung eines Denkens, das dank des dialektischen Verfahrens aus seinen falschen, weil notwendig unvollständigen Verdinglichungen herausgezerrt wird, die dazu führen, das es als autarkes Fürsich aufgefasst wird anstatt als Vermitteltes. Deshalb auch zeigt begriffliche Widersprüchlichkeit nicht etwa ein Unzureichendes der methodischen oder theoretischen Mittel, sondern die widersprüchliche Konstitution der Gegenstände, weshalb Adorno anführen kann, „, $[d]$ ie aporetischen Begriffe der Philosophie [seien] Male des objektiv, nicht bloß vom Denken Ungelöste $[n]$ “" (ND, 156). ${ }^{5}$ Das führt jedoch gleichzeitig auch dazu, einzusehen, dass der Versuchung einer Auflösung

5 Dass die Gegenstände notwendigerweise widersprüchlich sind, rührt daher, dass sie immer zu sein trachten, was sie zugleich notgedrungen nicht sind. In seiner Vorlesung zu negativer Dialektik betont Adorno, dass es sich um dieselbe Widersprüchlichkeit handelt, die auch die Gesellschaft kennzeichnet. Das hat seinen Grund darin, dass der Widerspruch vom Vollzug identifizierenden Denkens herrührt, das begründet ist ,in dem Prinzip der Identität: in dem immanenten Bestreben allen Geistes, sein Anderes, das was an ihn herangetragen wird oder worauf er stößt, sich 
der Widersprüche in Hegelscher positiver Negation zu widerstehen ist. Denn „, $[d]$ ass die Negation der Negation die Positivität sei, kann nur verfechten, wer Positivität, als Allbegrifflichkeit, schon im Ausgang präsupponiert“ (ND, 162), wer also m.a.W. die Identität, die es eigentlich erst auszuweisen gälte, a priori immer schon gesetzt hat, und sei es implizit. Stattdessen ist es an „[d]ialektische $[r]$ Erkenntnis [...], der Inadäquanz von Gedanke und Sache nachzugehen; sie an der Sache zu erfahren" (ND, 156). Das meint jedoch gleichzeitig auch, dass der Widerspruch nicht einfach immer schon vorhanden ist, sich nicht unmittelbar gibt, sondern selbst der subjektiven Vermittlung bedarf. In der Tat stellt Adorno fest, und vielleicht liegt auch das moralische Moment von Dialektik schon hier angezeigt, dass

[d]er dialektische Widerspruch [...] nicht schlechthin [,ist'], sondern [...] seine Intention - sein subjektives Moment - daran [hat], dass er das [Leiden der Menschen] nicht sich ausreden lässt; in [...] [ihrer Intention] geht Dialektik aufs Verschiedene. Philosophisch bleibt die dialektische Bewegung als Selbstkritik der Philosophie. (ND, 156) ${ }^{6}$

\section{Nichtidentisches und das Denken in Konstellationen}

Da weiter oben schon eine erste Definition des Nichtidentischen zumindest umrissen worden ist, soll es hier nur noch einmal darum gehen, diesen für das adornitische Denken zentralen Begriff ein wenig strukturierter darzustellen. In der Tat ist es für Adorno wesentlich, dass alles, „, $[w]$ as ist, [...] mehr [ist], als es ist“ (ND, 164). Dieses ,Mehr’ nun ist nicht hinzugefügt, sondern dem Gegenstand „immanent" (ND, 164), da es der jeweils singuläre Überschuss ist, den Denken (verstanden als das vernünftige Verfahren, das Gegenstände mit Begriffen identifiziert) nicht begrifflich fassen kann. In dieser Hinsicht spricht Adorno vom Nichtidentischen als der „eigene[ $n]$ Identität der Sache gegen ihre Identifikation“ (ND, 164). Das bedeutet jedoch gleichzeitig im Umkehrschluss auch, dass vom Standpunkt

gleichzumachen und in seinen Herrschaftsbereich hineinzuziehen“ Adorno: Vorlesung über Negative Dialektik, op. cit., S. 21.

6 Zum Unterschied der Position Adornos mit der Hegelschen Konzeption sei auch dieses Zitat angeführt, das noch einmal verdeutlicht, in welchem Maße das (negativ) dialektische Denken immer gleichzeitig auch moralisch ist: „Während fraglos die Konstruktion seines [Hegels] Systems ohne jenes Prinzip [der positiven Negation der Negation] zusammenstürzte, hat Dialektik ihren Erfahrungsgehalt nicht am Prinzip sondern am Widerstand des Anderen gegen die Identität; daher ihre Gewalt. In ihr steckt auch Subjekt, soweit dessen reale Herrschaft die Widersprüche erzeugt, aber diese sind ins Objekt eingesickert. Dialektik rein dem Subjekt zurechnen, den Widerspruch gleichsam durch sich selbst wegschaffen, schafft auch die Dialektik weg, indem sie zur Totalität ausgeweitet wird. Sie entsprang bei Hegel im System, hat aber nicht ihr Maß an ihm." (ND, 163) 
des begreifenden Verstandes aus notgedrungen das Gegenteil gelten muss, insofern das Nichtidentische sich dem Instrument des Begreifens, dem Begriff, verweigert. Deshalb „[erweist sich $d]$ as Innerste des Gegenstandes [...] als zugleich diesem auswendig" (ND, 164). Dieser Widerspruch rührt nun daher, dass Denken traditionell Allgemeines und Wesen zusammengedacht hat, wo beide eigentlich zu trennen wären. ${ }^{7}$ Dass beide Termini nicht notwendig zusammenfallen, sondern im Gegenteil sich ausschließen, bzw. in Konflikt miteinander sich befinden können, zeigt sodann ein Denken an, das sich in die Sachen versenkt, um sie in ihrer Eigenart zum Sprechen zu bringen, anstatt sie mit dem Allgemeinen naiv zu verwechseln. ${ }^{8}$

Doch wie kann Nichtidentisches gedacht werden, wenn es eben nicht begrifflich fassbar gemacht werden kann, Denken jedoch notwendigerweise immer begrifflich sein muss? Um Nichtidentisches zu erreichen, es ausdrücken zu können, und somit begrifflich zu enthüllen, macht sich Adorno den Umstand zunutze, dass Sprache „kein bloßes Zeichensystem für Erkenntnisfunktionen“ (ND, 164) ist. Gegen einen falschen Zwang zur leeren Abstraktion „von den Begriffen im Stufengang zum allgemeineren Oberbegriff“, lässt Adorno „sie in Konstellation treten“ (ND, 164). Er macht sich also zunutze, dass Sprache zuallererst Verhältnisse ausdrückt, und nicht einzelne, nebeneinander existierende Begriffe. Dieses Aufzeigen der Verhältnismäßigkeiten eines Sachverhaltes bringt sodann Letzteren ans Tageslicht, auch wenn er selbst nicht direkt begrifflich gefasst werden kann. Daher beschreibt Adorno das Denken in Konstellationen dank der Relationalität von Sprache wie folgt:

Wo sie wesentlich als Sprache auftritt, Darstellung wird, definiert sie nicht ihre Begriffe. Ihre Objektivität verschafft sie ihnen durch das Verhältnis, in das sie die Begriffe, zentriert um eine Sache, setzt. Damit dient sie der Intention des Begriffs, das Gemeinte ganz auszudrücken. Konstellationen allein repräsentieren, von außen, was der Begriff im Innern weggeschnitten hat, das Mehr, das er sein will so sehr, wie er es nicht sein kann. Indem die Begriffe um die zu erkennende Sache sich versammeln, bestimmen sie potentiell deren Inneres, erreichen denkend, was Denken notwendig aus sich ausmerzte. (ND, 164-165)

Im Gegenteil zu einem traditionellen instrumentellen Denkens, das Sachverhalte lediglich durch Identifizierung angleichen möchte, und sie somit in Wahrheit

7 Zu diesem Schluss kommt in der Tat Adorno, wenn er feststellt: „Dahin geleitet denkende Insistenz vorm Einzelnen, als auf dessen Wesen, anstatt auf das Allgemeine, das es vertrete.“ (ND, 164)

8 So führt Adorno aus: „Kommunikation mit Anderem kristallisiert sich im Einzelnen, das in seinem Dasein durch sie vermittelt ist.“ (ND, 164) 
weniger erkennt, denn vielmehr unter ein ihnen Verschiedenes subsumiert, geht es hierbei also darum, dem unnennbaren Einzelnen mittels des sprachlichen Ausdrucks dazu zu verhelfen, Sichtbarkeit zu erlangen, und zwar für sich, und nicht verkrüppelt als einfaches Signum eines Allgemeinen. ${ }^{9}$

\section{Zweiter Teil. Die moralphilosophische Antinomie und ihre Überwindung im Widerspruch.}

\section{Hinzutretendes und Impuls}

In Negative Dialektik widmet Adorno bekanntlich sein erstes Modell einer Kritik der Kantischen Freiheitslehre, gegen die er seine eigene Theorie moralischen Handelns ins Feld führt. Er wendet sich somit gegen eine ihm zufolge in der Moderne vorherrschende „Moralkonzeption, die inhaltlich durch die Idee der Gleichheit und formal durch die Struktur von Gesetz und Gewissen bestimmt ist “ ${ }^{10}$ In der Tat kritisiert Adorno an der Kantischen Konzeption eines Sittengesetzes dessen „repressiven Aspekt“ (ND, 257). Denn wenn das Subjekt sich dieses Gesetz in Freiheit selbst gibt und sodann aus Freiheit befolgt, dann ist eine in die „Autonomie beigemischte Heteronomie" (ND, 258) unvermeidlich. Vergeblich ist in der Tat eine Freiheitslehre, die sich in ihrer Ausführung zugleich immer auch wieder kassiert, da „[d]ie Nötigung, die laut Kant vom kategorischen Imperativ ausgeht, [...] der Freiheit [widerspricht], die in ihm als ihrer obersten Bestimmung sich zusammenfassen soll“ (ND, 258). So konstatiert Adorno bezüglich der formalen Kantischen Lehre, dass

9 Natürlich kann ,für sich' hier nicht meinen, dass das Nichtidentische keine Beziehung zu Allgemeinem unterhielte, sondern lediglich, dass nur ein solches Denken in Konstellationen es erschließen kann als das, was es ist. Siehe auch folgendes Zitat Adornos zum Verhältnis des Nichtidentischen und des Begriffs: „Jene Supposition [das alles Geist wäre] ist nicht zu retten. Wohl aber transzendiert das in keinen vorgedachten Zusammenhang Auflösliche als Nichtidentisches von sich aus seine Verschlossenheit. Es kommuniziert mit dem, wovon der Begriff es trennte. Opak ist es nur für den Totalitätsanspruch der Identität; seinem Druck widersteht es. Als solches jedoch sucht es nach dem Laut. Durch die Sprache löst es sich aus dem Bann seiner Selbstheit. Was am Nichtidentischen nicht in seinem Begriff sich definieren lässt, übersteigt sein Einzeldasein, in das es erst in der Polarität zum Begriff, auf diesen hinstarrend, sich zusammenzieht. Das Innere des Nichtidentischen ist sein Verhältnis zu dem, was es nicht selber ist und was seine veranstaltete, eingefrorene Identität mit sich ihm vorenthält. Zu sich gelangt es erst in seiner Entäußerung, nicht in seiner Verhärtung $[\ldots]^{“}(\mathrm{ND}, 165)$.

10 Menke, Christoph: „Modell 1: Freiheit. Zur Metakritik der praktischen Vernunft II“, in: Honneth, A. und Menke, C. (Hrsg.), Klassiker Auslegen: Negative Dialektik, Akademie Verlag, Berlin 2006, S. 151-168. 
[d]ie Antinomik der Kantischen Freiheitslehre [...] darin sich [zuspitzt], dass ihr das Sittengesetz unmittelbar für vernünftig gilt und für nicht vernünftig; vernünftig, weil es sich auf reine logische Vernunft ohne Inhalt reduziert; nicht vernünftig, weil es in seiner Gegebenheit zu akzeptieren, nicht weiter zu analysieren sei [...]. (ND, 258)

So schlägt schlussendlich die Freiheitslehre Kants in ihr repressives Gegenteil um und „Ratio wird zur irrationalen Autorität“ (ND, 258).

Ein weiteres Problem der Kantischen Konzeption ist darüber hinaus der Umstand, dass sich diese Lehre nicht so sehr auf das Problem der moralischen Praxis bezieht, als vielmehr auf das sittliche Gesetz, dass aber wiederum die praktische Anwendung des letzteren nicht so durchschaubar ist, wie Kant es unterstellt ${ }^{11}$. Das hat zwar einerseits den Vorteil, dass Kant nicht in Versuchung gerät, positive Handlungsanweisungen zu formulieren ${ }^{12}$, führt aber andererseits zu einer „Entgegenständlichung", welche schlussendlich in eine Moral der simplen und alleinigen „Kontemplation“ (ND, 235) mündet, die im Hinblick auf moralische Handlungen sich als nutzlos erweist, da der Sprung von der Vernunft in die Welt versperrt bleibt. ${ }^{13}$ So fasst denn auch Menke zusammen: „Wird die Vernunft allein als Vermögen der Gesetzgebung verstanden, dann kann sie [...] nicht das Vermögen zur moralischen Entscheidung und Handlung umfassen“.14

Gegen diese moralphilosophische Konzeption führt Adorno nun seine eigene Theorie an, die auf einem Vermittelnden fußt, das er "das Hinzutretende“ nennt. Dieses ist für ihn dasjenige Moment, das zu einer (moralischen) Handlung erst führt. In der Tat gehört es nach Adorno zur menschlichen Freiheit, dank eines „Ruck[s]“ neue „Kausalkette[n]“ (ND, 226) anzustoßen. Dieser Ruck nun ist das Hinzutretende, das die "philosophische Tradition wieder nur als Bewusstsein" (ND, 226) versteht. Wenn das jedoch der Fall wäre, so wäre nicht einsichtig, warum eben das Hinzutretende nun den Sprung aus der Vernunft heraus schaffen kann, den diese vorher nicht vollbringen konnte. ${ }^{15}$ Im Gegensatz zu der traditionellen

11 Hierzu auch Adorno: Probleme der Moralphilosophie, Suhrkamp, Frankfurt a. M. 2010 [1963], S. 233ff., und Menke: Modell 1, art. cit.

12 Hierzu schreibt Adorno: „Während er keine positive Kasuistik des zu Tuenden an die Hand gibt, verhindert er human den Missbrauch inhaltlich-qualitativer Differenzen zugunsten des Privilegs und der Ideologie." (ND, 235)

13 In diesem Zusammenhang stellt Adorno fest: „Die Kritik der praktischen Vernunft bewegt sich im Verblendungszusammenhang. Ihr schon dient Geist als Surrogat der Handlung, die da nichts sein soll, als der schiere Geist." (ND, 236)

14 Menke: Modell 1, art. cit.

15 Völlig zu Recht weist Menke auch darauf hin, dass das ,Hinzutretende’ nicht als „Motiv“ zu interpretieren ist, dient es doch gerade dazu, auf eben dieses in der Moralkonzeption nicht zurückgreifen zu müssen. Ibid. 
Auffassung begreift Adorno das Hinzutretende als einen „Impuls“, der, obwohl dem Bewusstsein nicht äußerlich, sich doch in diesem nicht erschöpft, sondern zugleich, "intramental und somatisch in eins", auch ein Somatisches besitzt. Dergestalt "treibt [er] über die Bewusstseinssphäre hinaus, der er doch auch angehört" (ND, 227228). Er ist somit rationalistisch und empiristisch zugleich, und überwindet diese Gegensätze dadurch. So schreibt auch Menke als Erläuterung des Hinzutretenden:

Entgegen dieser Zerreißung in zwei unverbundene Elemente (die im Gegensatz von Rationalismus und Empirismus zum Ausdruck kommen), soll der Impulsbegriff mithin auf den Punkt hinweisen, an dem in allem Handeln und Wollen der Gegensatz von Freiheit und Natur (,immer schon') überwunden, ja, das ,Phantasma [...] einer Versöhnung von Geist und Natur' (227) walten muss, damit Handeln und Wollen überhaupt möglich sind. ${ }^{16}$

In diesem Zitat scheint durch, dass das Hinzutretende als die objektive Konstitution im Subjekt verstanden werden muss. ${ }^{17}$ Mit anderen Worten, es ist das Objektive im Subjekt, dass die subjektive Sphäre sowohl übersteigt, als auch fundiert. Für das Hinzutretende gilt, was Adorno zur Empfindung ausführt:

Die Empfindung, crux aller Erkenntnistheorie, wird erst von dieser, im Widerspruch zu ihrer eigenen vollen Beschaffenheit, welche doch die Rechtsquelle der Erkenntnis sein soll, in eine Tatsache des Bewusstseins uminterpretiert. Keine Empfindung ohne somatisches Moment. Insofern ist ihr Begriff, gegenüber dem, was er angeblich subsumiert, dem Verlangen eines autarkischen Zusammenhangs aller Stufen der Erkenntnis zuliebe verbogen. (ND, 193)

\section{Von der Möglichkeit einer Sphäre des autonom Moralischen}

Der so gefasste Begriff des Hinzutretenden erlaubt es nun Adorno, die Vermittlung von Subjektivem und Objektivem in der Problemstellung der Moral anders zu erwägen, als es Kant getan hat. Moralische Praxis bedarf auch nach Adorno des fortschrittlichsten "theoretischen Bewusstseins" (ND, 228), soll sie nicht in Blindheit, d.h. in Gewalt umschlagen. ${ }^{18}$ Aber sie

16 Ibid.

17 Hierzu s.a. Kern, Andrea: „Negative Dialektik. Begriff und Kategorien I“, in Honneth und Menke, Klassiker Auslegen, op.cit., S. 49-69.

18 In der Tat kann nur Theorie gewährleisten, dass die Handlung nicht in blinde, und somit gewalttätige Handlung umschlägt. Das hat Adorno im Blick, wenn er schreibt: „Wahre Praxis, der Inbegriff 
bedarf [eben] auch eines Anderen, in Bewusstsein nicht sich Erschöpfenden, Leibhaften, vermittelt zur Vernunft und qualitativ von ihr verschieden. Beide Momente werden keineswegs getrennt erfahren; doch hat die philosophische Analyse das Phänomen derart zurechtgestutzt, dass es danach, in der Sprache der Philosophie, gar nicht anders kann ausgedrückt werden, als wie wenn zu Rationalität ein Anderes addiert würde. (ND, 229)

Jedoch hat dieses Andere ein Moment des Eruptiven. Es ist ebenso zwingend, wie es zugleich eine Zäsur darstellt. Im Angesicht des Unerträglichen drängt es sich dem Individuum als leibhaftige Erfahrung auf. Jedoch kann keine positive Norm von diesem Impuls abgeleitet werden, da jene wiederum en définitive in autoritäre Unfreiheit umschlüge. Deshalb kann Adorno schreiben: „[w]ahr sind die Sätze als Impuls, wenn gemeldet wird, irgendwo sei gefoltert worden. Sie dürfen sich nicht rationalisieren; als abstraktes Prinzip gerieten sie sogleich in die schlechte Unendlichkeit ihrer Ableitung und Gültigkeit“ (ND, 281). Dass der Impuls, vom Objekt gezeitigt, sich aufdrängt, ist Zeichen seiner in der Vermittlung unmittelbar sich offenbarenden Wahrheit. Gleichzeitig deutet er jedoch immer auch die notwendige Fehlbarkeit der Moral an, von der Adorno schreibt:

Im Gegensatz zu seinem Pharisäismus verurteilt das irrationale Moment des Willens alles Moralische prinzipiell zur Fehlbarkeit. Moralische Sicherheit existiert nicht; sie unterstellen wäre bereits unmoralisch, falsche Entlastung des Individuums von dem, was irgend Sittlichkeit heißen dürfte. (ND, 241)

Diesen Nachteil - der zwingend ist, da moralische Sicherheit wiederum eine ungerechte, weil kategoriale, und somit vereinheitlichende Setzung wäre - wiegt auf, dass ein solcher Impulsbegriff das Problem der heteronomen Motivation löst, da dieses ganz im Impuls als „Grund“ der „moralische[ $n]$ Erkenntnis“19 aufgeht. Gleichzeitig ist das moralisch handelnde Subjekt in der Situation insofern autonom, als dass es durch die impulsive Vermittlung seiner objektiven Verdinglichung entledigt ist. Denn es wird dank des Impulses sichergestellt, dass eben keine äußere, allgemeine und somit abstrakte Norm zur Beurteilung der jeweiligen Situation und zur Handlungsentscheidung zugrunde gelegt wird. Anders ausgedrückt kann die drohende Verdinglichung dadurch vermieden werden, dass das Subjekt dank

von Handlungen, welche der Idee von Freiheit genügen, bedarf zwar des vollen theoretischen Bewusstseins. Der Dezisionismus, der die Vernunft im Übergang zur Handlung durchstreicht, liefert diese dem Automatismus der Herrschaft aus: die unreflektierte Freiheit, die er sich anmaßt, wird zum Knecht totaler Unfreiheit.“ (ND, 228)

Menke: Model 1, art. cit. 
des Hinzutretenden nicht setzend agiert, sondern dem Einzelnen gegenüber der Allgemeinheit den Vorzug gibt. Das meint die Feststellung Menkes, „[ $s]$ ich moralisch zu entscheiden, besteh [e] darin, dem Ruf zu folgen, der von einer Situation her an das Subjekt ergeht“. ${ }^{20}$

Allerdings muss in diesem Zusammenhang erwähnt werden, dass Menke Adornos Konzeption des Moralischen in die Nähe einer Tugendethik zu rücken scheint. ${ }^{21}$ Dem können wir nicht folgen. In der Tat geht es hier wohl weniger um „die Fähigkeit und die Bereitschaft zur Verwirklichung sittlicher Werte in Gesinnung und Haltung "22, als vielmehr um eine praktische Philosophie, in welcher das Gelingen moralischer Handlungen wesentlich davon abhängt, ob der Agent gewissermaßen in subjektiver ,Entdinglichung' in der Lage ist, seine Subjektivität kritisch reflektierend zu erkennen. Während eine Tugendlehre gleichzeitig eine spezifische Prädisposition zur Bedingung macht (der Tugendhafte muss gleichzeitig um das Gute wissen und es wollen), die folglich nicht alle haben können, hat Adorno immer wieder betont, dass er dem von Kant in Was ist Aufklärung? formulierten aufklärerischen Gedanken folgt, wonach alle Menschen vernünftig seien, und es nurmehr auf das Bestreben jedes Einzelnen ankomme, seine Unmündigkeit abzuschütteln. Somit geht es hier folglich weniger um Tugend, als vielmehr um Kritik.

\section{Leiden, das Hinzutretende in moralischer Hinsicht}

Vom Somatischen in der Erkenntnis ist im Hinblick auf das ,Hinzutretende' schon die Rede gewesen. Hierzu gehört nun auch das Leid. In der Tat kann man letzteres

20 Menke: Model 1, art. cit. In der Tat fügt Menke hier, in Anlehnung an Adornos Ausführungen aus Probleme der Moralphilosophie, op. cit., hinzu: „So ist die ,Einschränkung des Gefühls der Souveränität' des Subjekts die ,Bedingung des Moralischen selber"'. So wird gleichzeitig verständlich, wieso Adorno in dieser Vorlesung auf dem „Nicht-sich-selber-Setzen“ (Ibid., S. 251) als dem heute noch möglichen Moralischen beharrt, und was dies aus theoretischer Sicht bedeutet.

21 Cf. Menke: Model 1, art.cit. „In der damit angezielten konstitutiven Verknüpfung von Vernunft und Natur, Mentalem und Somatischem gleicht Adornos Begriff des Impulses gegenwärtigen Theorien, die [...] im Rückgriff auf Motive des Aristotelischen Tugendbegriffs auch für die moralische Erkenntnis den ,Vorrang des Objekts' (Adorno) geltend machen."

22 So lautet die Definition von Tugend in den Moral- und Wertlehren des zwanzigsten Jahrhunderts, zusammengefasst im Artikel „Tugend“, in: Regenbogen, Arnim und Meyer, Uwe: Wörterbuch der philosophischen Begriffe, Felix Meiner, Hamburg 2013, S. 675-676. Der Eintrag zeigt des Weiteren anschaulich, welche Bedeutungsveränderungen der Begriff im Laufe der Geschichte durchwandert hat, wobei im Zusammenhang mit unserer Problemstellung zu betonen ist, dass die Tugend doch im Allgemeinen eine persönliche Disposition zur guten Lebensführung ausweist, deren Vorteil sich darin ausdrückt, dass das Gemeinwohl von der Verhaltensäußerung des tugendhaften Einzelnen profitiert. Somit scheint uns der Begriff der Tugend eine Normativität zumindest zu implizieren, die Adorno in der Problematisierung des Hinzutretenden gerade ob der durch sie evozierten Heteronomie zu umgehen trachtet. 
verstehen als das moralisch Hinzutretende in seiner gesellschaftlichen Dimension. So spricht Adorno in diesem Zusammenhang davon, dass „, $[d]$ as leibhafte Moment [...] der Erkenntnis [anmeldet], dass Leiden nicht sein, dass es anders werden solle" (ND, 203). Das Leiden überbringt somit in seiner Anklage auch die utopische Verheißung eines versöhnten Zustands, in welchem es nicht mehr wäre. Das zielt immer auf die Totalität in Form der Gesellschaft. So stellt Adorno unmissverständlich fest:

Die Abschaffung des Leidens, oder dessen Milderung hin bis zu einem Grad, der theoretisch nicht vorwegzunehmen, dem keine Grenze anzubefehlen ist, steht nicht bei dem Einzelnen, der das Leid empfindet, sondern allein bei der Gattung, der er dort noch zugehört, wo er subjektiv von ihr sich lossagt und objektiv in die absolute Einsamkeit des hilflosen Objekts gedrängt wird. (ND, 203)

Eine solche Aufhebung des Leids beinhaltet notwendig eine kritische Theorie, die in negativer Dialektik das Nichtidentische von der ihm angetanen Gewalt erlöst, und ihm sein Recht zuteil werden lässt. Das schwingt mit, wenn Adorno schreibt,

[e]ine solche Einrichtung [der Gesellschaft] hätte ihr Telos an der Negation des physischen Leidens noch des letzten ihrer Mitglieder, und der inwendigen Reflexionsformen jenes Leidens. Sie ist das Interesse aller, nachgerade einzig durch eine sich selbst und jedem Lebenden durchsichtige Solidarität zu verwirklichen. (ND, 204)

So wird deutlich, dass die praktische Philosophie Adornos in ihrem Anspruch nie lediglich partikular sein kann. Dies ist ein Ergebnis nicht so sehr ihrer Negativität, denn vielmehr ihrer grundsätzlichen Dialektik. Denn das Zentrum einer solchen Philosophie bildet demzufolge der Anspruch, die dialektischen Vermittlungen, in denen sich ihre einzelnen Elemente bedingen, denkend nachzuvollziehen, ohne dass dabei jemals einfach eingehalten werden könnte. ${ }^{23}$

23 Zur Veranschaulichung der Bedeutung dieser Feststellung kann das Ergebnis herangezogen werden, zu dem F. Freyenhagen in seiner Arbeit zur praktischen Philosophie Adornos gelangt. Freyenhagen schlägt vor, dass drei Faktoren für ein weniger falsches Leben durch Widerstand vonnöten wären: "critical reflection ", " a sense of fallibility and modesty ", und "the identification [sic.] and solidarity with others » Freyenhagen, Fabian: Adorno's practical philosophy, Cambridge University Press, Cambridge 2013, S. 175. So leicht das erste Kriterium eingesehen werden kann, so schwierig erscheinen uns die beiden anderen. Grundsätzlich scheint es nämlich auch hier wieder, als wäre es Adorno nicht so sehr um Tugend zu tun, als vielmehr um den dialektischen Moment des Nichtsich-selber-Setzens (Adorno: Probleme der Moralphilosophie, op. cit., S. 251). Ebenso handelt es sich weniger um eine identification, ein im Zusammenhang mit der adornitischen Philosophie im Übrigen mehr als problematischer Terminus, selbst wenn er hier im Zusammenhang mit der leidenden Kreatur verwendet wird, als vielmehr um ein mimetisches Erkennen. Das Problem scheint somit 


\section{Dritter Teil. Von der Hoffnung auf Richtiges im Falschen.}

\section{Das notwendige Moment der Freiheit im Falschen}

Eine solche Dialektik hat Auswirkungen auf das Denken von Freiheit. Denn auch das Problem der Freiheit muss im Widerspruch zweier ineinander vermittelter Entitäten gedacht werden, den Individuen einerseits und der Gesellschaft andererseits. Deshalb ist es Adorno zufolge auch ein klassischer Fehler, Freiheit einzig vom Subjekt her zu denken, das darüber hinaus noch als von der Gesellschaft abgespalten betrachtet wird. Dabei wird nämlich nicht berücksichtigt, dass

$[d]$ as vermeintlich ansichseiende Subjekt $[\ldots]$ in sich vermittelt [ist] durch das, wovon es sich scheidet, den Zusammenhang aller Subjekte. Durch die Vermittlung wird es selber das, was es seinem Freiheitsbewusstsein nach nicht sein will, heteronom. Auch wo Unfreiheit positiv unterstellt wird, sucht man ihre Bedingungen, als solche einer immanent geschlossenen psychischen Kausalität, in dem abgespaltenen Individuum auf, das wesentlich kein derart Abgespaltenes ist. (ND, 213)

Freiheit ist demgegenüber auch kein Zustand, der, einmal erreicht, fortbesteht. Das unterschlüge, dass Freiheit in sich antinomisch ist, Freiheit immer Unfreiheit mit sich führt. ${ }^{24}$ Trotzdem ist die Idee der Freiheit notwendig, weil sie die „Integration der Gesellschaft, die den Subjekten widerfährt, als wäre sie unwiderstehlich“ (ND, 215) als eben eine solche entlarvt. Zugleich besteht hier auch eine Gefahr, denn die gesellschaftliche Integration wirkt auch auf den „Charakter oder Wille[ $n]$, das potentielle Organ der Freiheit“ (ND, 216), um ihn zu einem Herrschaftsinstrument zu formen. Das verlangt nach einer Kritik des Agens von Freiheit. Deshalb braucht es eine kritische Theorie, denn

zu sein, dass Freyenhagen die moralische Situation in nicht-dialektischer Weise denkt. Deshalb vermag sein Ansatz nicht, die dialektische Dynamik auszudrücken, die auf das Subjekt wirkt, und die in moralischer Hinsicht eine Attitüde der nicht-Setzung verlangt. Dabei ist es ebendiese dialektische Dynamik in der Erfahrung, die ein ebenso konkretes wie universelles Moment hervorruft, und somit das moralische Erkennen einer Situation erst ermöglicht. J. Butler wiederum hat in ihrer Analyse der notwendigerweise aporetischen moralischen Handlung die gegenseitige dialektische Bedingtheit der situativen Bestimmungen treffend erläutert. Ihr zufolge führt erst diese Bedingtheit dazu, dass kein begriffliches Moment endgültig gesetzt werden kann, ohne dass dadurch ebendieses Moment in Unwahrheit umschlüge (Butler, Judith: Kritik der ethischen Gewalt: Adorno-Vorlesungen 2002, Suhrkamp, Frankfurt a. M. 2014, S. 145). Dieser Umstand begründet die notwendige Unsicherheit des moralischen Moments.

24 Schon alleine in der Feststellung: „Die Souveränität des Gedankens, der vermöge seiner Freiheit auf sich als auf sein Subjekt sich zurückwendet, zeitigt auch den Begriff Unfreiheit. Beides ist kein einfacher Gegensatz sondern ineinander." (ND, 219) 
Identität des Selbst und Selbstentfremdung begleiten einander von Anbeginn; darum ist der Begriff Selbstentfremdung schlecht romantisch. Bedingung von Freiheit, ist Identität unmittelbar zugleich das Prinzip des Determinismus. Wille ist soweit, wie die Menschen sich zum Charakter objektivieren. (ND, 216)

Das zeigt an, dass eine Theorie der Freiheit zwingend auch die Rücksichtnahme auf die Vermittlung von Subjekt und Objekt beinhaltet. ${ }^{25}$ Nun ist festzustellen, dass die Gesellschaft aufgrund ihrer instrumentellen Identifizierung keine freie ist. Daher wäre das Moment der Möglichkeit von Freiheit im Individuum zu verorten, auch wenn zuvor dessen heteronome Ableitung aus objektiver Gesellschaft eingesehen worden ist. ${ }^{26}$ Trotzdem bleibt festzustellen:

Das principium individuationis ist [...] keineswegs das metaphysisch Letzte und Unabänderliche, und darum auch nicht die Freiheit; diese vielmehr Moment im doppelten Sinn: nicht isolierbar sondern verflochten, und einstweilen stets nur ein Augenblick von Spontaneität, geschichtlicher Knotenpunkt, verstellt unter den gegenwärtigen Bedingungen. (ND, 218)

Das Individuum ist also nur insofern Träger der Möglichkeit von Freiheit, als dass es sich in theoretischer Reflexion zumindest negativ vom gesellschaftlichen Verblendungszusammenhang befreien kann; gleichzeitig entdeckt es sich dadurch als von der Gesellschaft Produziertes, und somit als Agent der Unfreiheit insofern, als

25 So stellt Adorno im Hinblick auf die traditionelle Philosophie fest: „Eskamotiert wird a priori die Wechselwirkung von Subjekt und Objekt, Spontaneität schon durch die Methode ausgeschlossen [...]“ (ND, 216). Und weiter unten schreibt er: „So wenig ist das Subjekt die ,Sphäre absoluter Ursprünge', als die es sich philosophiert; noch die Bestimmungen, kraft deren es seine Souveränität sich zuspricht, bedürfen immer auch dessen, was ihrem Selbstverständnis nach bloß ihrer bedürfen soll. Über das am Ich Entscheidende, seine Selbstständigkeit und Autonomie kann nur geurteilt werden im Verhältnis zu seiner Andersheit, zum Nichtich. Ob Autonomie sei oder nicht, hängt ab von ihrem Widersacher und Widerspruch, dem Objekt, das dem Subjekt Autonomie gewährt oder verweigert; losgelöst davon ist Autonomie fiktiv." (ND, 222)

26 So schreibt Adorno: „Die Antinomie zwischen der Determination des Individuums und der ihr kontradiktorischen gesellschaftlichen Verantwortung ist kein falscher Gebrauch der Begriffe sondern real, die moralische Gestalt der Unversöhntheit von Allgemeinem und Besonderem. " (ND, 261) $\mathrm{Zu}$ der Feststellung, das Moment der Freiheit läge heute trotz Allem im Individuum, passt im Übrigen auch, was Adorno in seiner Vorlesung Zur Lehre der Geschichte und der Freiheit ausführt, nämlich dass das Kantische Diktum des radikal bösen freien Menschen umzudrehen sei, weil „noch keine Welt hergestellt ist, in der sie nicht mehr radikal böse zu sein brauchten und in der infolgedessen also die Unfreiheit, in deren Bann sie selber sind, gebrochen wäre." Adorno: Zur Lehre von der Geschichte und von der Freiheit, Suhrkamp, Frankfurt a. M. 2006 [1964/1965], S. 280. 
dass es an der Identifizierung im gesellschaftlichen Zusammenhang teilhat. ${ }^{27}$ Das schwingt in dem berümten Diktum aus den Minima Moralia mit, es gebe „kein richtiges Leben im falschen“. ${ }^{28}$ Wenn dieser Satz auch vordergründig auf die gewordene Unmöglichkeit des Wohnens in bürgerlicher Innerlichkeit bezogen ist, findet sich hier nichtsdestoweniger zugleich ein Rat, der analog in den moralphilosophischen Überlegungen aufzeigbar ist, wenn Adorno darauf drängt, man solle das Verhältnis zu den Gütern aufheben, so dass es in einem Zustand der Schwebe verweile. ${ }^{29}$ In der Tat zeigt dies an, worin Adorno in der modernen Gesellschaft die einzig mögliche Form von Freiheit verortet, nämlich darin, „dass das richtige Leben heute in der Gestalt des Widerstandes gegen die von dem fortgeschrittensten Bewusstsein durchschauten, kritisch aufgelösten Formen eines falschen Lebens bestünde“. 30

\section{Widerstand als praktische bestimmte Negation}

Das Moment der Freiheit residiert somit heute zuvorderst im in bestimmter Negation begründeten, d.h. sich nicht selbst positiv setzenden Widerstand, und kann daher als ein Ablehnen des Mitmachens bezeichnet werden. ${ }^{31}$ Das wird Adorno nicht müde zu unterstreichen. So stellt er zum Beispiel in Negative Dialektik fest,

$[f]$ rei wäre erst, wer keinen Alternativen sich beugen müsste, und im Bestehenden ist es eine Spur von Freiheit, ihnen sich zu verweigern. Freiheit meint Kritik und

27 In einem anderen, weil vordergründig epistemologischen Kontext, der jedoch auch die ethische Sphäre notgedrungen bedingt, schwingt diese fein verwobene Dialektik in der Feststellung mit, der Gedanke, der aus Freiheit auf sich selbst reflektiert, sei gerade in dieser Bewegung Beweis seiner identifizierenden Unfreiheit ( $c f$. ND, 219). Das dem so ist und dies des Weiteren auch für die ethische Fragestellung zureicht, zeigen die Gedanken Adornos zum Ende seiner Vorlesung Zur Lehre von der Geschichte und von der Freiheit, op. cit., S. 370 an.

28 Adorno: Minima Moralia, Suhrkamp, Frankfurt a. M. 2003 [1951], S. 43.

29 So stellt Adorno fest : „[d] as beste Verhalten all dem [zeitgenössischen Design] gegenüber scheint noch ein unverbindliches, suspendiertes" Ibid.

30 Adorno: Probleme der Moralphilosophie, op. cit., S. 249.

31 Cf. Ibid. In der Tat vermag nur ein solcher Widerstand der bestimmten Negation einerseits den gesellschaftlichen Bann zu durchbrechen, und andererseits zu verhindern, dass eine kodifizierte Moral des abstrakten Rigorismus' blind befolgt würde, was die moralische Handlung zwangsläufig wieder ins Unrecht stürzte. Auch wenn hier nicht in gebührender Weise darauf eingegangen werden kann, beinhaltet eine solche, auf bestimmter Negation beruhende moralische Handlung nie nur die Kritik der Umstände, sondern immer zugleich auch eine kritische Reflexion auf die gesellschaftliche Bedingtheit des moralischen Subjekts. Hierzu $c f$. Ibid., S. 248-251. Nur dies ermöglicht schlussendlich das für die moralische Handlung notwendige „Nicht-sich-selber-Setzen“ (Ibid., S. 251) des moralischen Subjekts. 
Veränderung der Situationen, nicht deren Bestätigung durch Entscheidung inmitten ihres Zwangsgefüges. (ND, 225-226, Anm.) ${ }^{32}$

Doch so einleuchtend diese Feststellung scheinen mag, wirft sie zugleich die Frage auf, wonach sich die Negation, so bestimmt sie sein mag, zu richten habe? Diese Frage nach dem „Moment des Übergangs von dem richtigen Bewusstsein zu dem

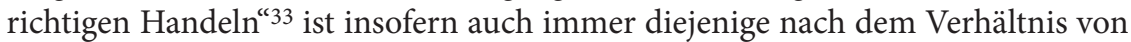
Theorie und Praxis. ${ }^{34}$

Adorno beantwortet dieses Problem mit der Tätigkeit des Hinzutretenden. Hierin berührt die in der bestimmten Negation sich artikulierende Freiheit mit dem der Vernunft inhärenten somatischen moralischen Impuls, der ihr bedeutet, wogegen sie sich in Widerstand zu äußern hat. Dieser Impuls ist es gleichzeitig auch, der anzeigt, in welchen Situationen es sich um Freiheit, und nicht etwa um unzulänglich durchschaute Unfreiheit handelt. Nicht von ungefähr erinnert denn auch Adornos nachfolgende Schilderung an die Erfahrung des Hinzutretenden als Ablehnung in einem So soll es nicht sein, wenn er schreibt,

[k]onkret [würde] Freiheit an den wechselnden Gestalten der Repression: im Widerstand gegen diese. Soviel Freiheit des Willens war, wie Menschen sich befreien wollten. Freiheit selbst aber ist derart mit der Unfreiheit verfilzt, dass sie von dieser nicht bloß inhibiert wird, sondern sie zur Bedingung ihres eigenen Begriffs hat. So wenig wie irgendein anderer einzelner, ist dieser als Absolutes auszusondern. (ND, 262)

So ist Freiheit als Widerstand denn „einzig in bestimmter Negation zu fassen, gemäß der konkreten Gestalt von Unfreiheit. Positiv wird sie zum Als ob“ (ND, 230), m.a.W. zu einer erneuten Setzung von Identischem über Nichtidentisches, und somit zur Unfreiheit. ${ }^{35}$ Eine solche Freiheit im Widerstand gegen die konkreten Formen der Unterdrückung eröffnet dadurch eine metaphysische Dimension, die

32 Diese Definition von Freiheit verweist natürlich auf den $\$ 100$ „Sur l'eau“, der den zweiten Teil der Minima Moralia beschließt, und in dem sowohl der utopische Gehalt, wie auch die in ihm angelegte Radikalität des Adornoschen Freiheitsbegriffs durchscheinen ( $c f$. Adorno: Minima Moralia, op. cit., S. 179). Für eine ausführlichere Besprechung dieses Stücks, $c f$. z. B. Düttmann, Alexander Garcia: So ist es. Ein philosophischer Kommentar zu Adornos, Minima Moralia', Suhrkamp, Frankfurt a. M. 2004, S. 59-62.

33 Adorno: Probleme der Moralphilosophie, op. cit., S. 166.

$34 \mathrm{Zu}$ diesem Problem in moralphilosophischer Hinsicht, $c f$. Ibid., und insbesondere die 11. Vorlesung vom 4.7.1963, S. 164-179.

35 Das bedeutet freilich auch, dass es zwar einerseits keinen abschließenden Begriff der Freiheit geben kann, sie aber zugleich unablässig in der bestimmten Negation zu formulieren ist, der notwendigerweise eine Kritik sowohl der gesellschaftlichen Verhältnisse einerseits, als auch der in ihnen befindlichen Individuen andererseits beizufügen ist. Das hat Adorno im Sinn, wenn er feststellt, 
der falschen Identifizierung deshalb zu entgehen vermag, weil sie sich subjektiv eben gerade nicht mehr selbst absolut setzt. Dies ermöglicht es sodann, einzusehen, dass eine solche Moralvorstellung zugleich eine metaphysische im Sinne negativer Dialektik ist. ${ }^{36}$ So kommt es nicht von ungefähr, dass Adornos metaphysische Überlegungen, die den letzten Teil von Negative Dialektik bilden, mit dem neuen kategorischen Imperativ, „daß Auschwitz sich nicht wiederhole“ (ND, 358), einsetzen. Hierbei handelt es sich wohl in moralischer Weise um die metaphysische Erfahrung unserer Zeit, und ihr ist nur gerecht zu werden, indem die Identifikation negativ dialektisch überwunden wird, während das Nichtidentische so zu seinem Recht kommt. Das ermöglicht eine neue Art von Metaphysik, von der Adorno erklärt:

Das Absolute jedoch, wie es der Metaphysik vorschwebt, wäre das Nichtidentische, das erst hervorträte, nachdem der Identitätszwang zerging. Ohne Identitätsthese ist Dialektik nicht das Ganze; dann aber auch keine Kardinalssünde, sie in einem dialektischen Schritt zu verlassen. Es liegt in der Bestimmung negativer Dialektik, dass sie sich nicht bei sich beruhigt, als wäre sie total; das ist ihre Gestalt von Hoffnung. (ND, 398)

\section{Reflexive Subjektivität und das befreite Ganze im Einzelnen}

Die angestellten moralphilosophischen Betrachtungen zeigen an, dass es Adorno zufolge unabdingbar ist, einen Begriff von Subjektivität zu formulieren, der durch seine Reflexion, welche auch die Berücksichtigung der hier vorgestellten Gedanken einschließt, imstande ist, sich von der unterdrückenden gesellschaftlichen Bedingtheit freizumachen. Im Licht dieser Forderung wird auch das vordergründig paradoxe Zitat Adornos begreiflich, wonach „,[i]m Kern des Subjekts [...] die objektiven Bedingungen [wohnen], die es um der Unbedingtheit seiner Herrschaft willen verleugnen muss und die deren eigene sind. Ihrer müsste das Subjekt sich entäußern. Voraussetzung seiner Identität ist das Ende des Identitätszwangs“ (ND, 277). Dass nur des Subjekts ,Identität’ mit seinem ,Identitätszwang’ aufräumen kann, meint hier, dass ihm das kritische Verfahren negativer Dialektik im Vollzug seine wirkliche Konstitution offenbart, und so das Subjekt sozusagen zu sich selbst

dass „, $[d]$ ie Frage nach der Freiheit [...] kein Ja oder Nein sondern Theorie [erheischt], die wie über die bestehende Gesellschaft so über die bestehende Individualität sich erhebt.“ (ND, 279)

Es sei hier nur am Rande erwähnt, dass das Folgende u.E. auch zu erklären vermag, warum die moralische Erfahrung immer zugleich eine metaphysische ist, insofern in ihrer Einzigartigkeit dem Subjekt das wahrhaft Absolute durchsichtig wird. Bezüglich der metaphysischen Erfahrung, cf. ND, 366ff. 
führt, was zugleich natürlich nicht im Sinne eines essentiellen Entfremdungskonzept zu verstehen ist.

Einzig dergestalt kann nach Adorno die identifizierende Verdinglichung überwunden werden, um sodann den bislang erstarrten Momenten ihre eigentliche Dynamik wiederzugeben. So stellt Adorno denn auch in seiner Vorlesung zur Negativen Dialektik fest:

Je mehr die Autonomie von Subjektivität kritisch sich einschränkt, sich ihrer als eines Vermittelten bewusst wird, desto bündiger die Verpflichtung, den Objekten jenen Vorrang zu lassen, der dem Gedanken das an Festigkeit einbringt, was er nicht in sich hat, dessen er bedarf, und ohne das nicht einmal jene Dynamik wäre, mit welcher Dialektik das Feste auflöst. ${ }^{37}$

Bis dahin gründet der übermächtige objektive gesellschaftliche Zwang in einem Subjekt, das sich naiv zum Herrscher über die Welt aufschwingt, während es in Wahrheit als bloße Handpuppe einem blinden Objektiven gehorcht.

Dagegen soll das Verfahren einer negativen Dialektik dazu dienen, die einzelnen Gegenstände aufzuschließen und zum Sprechen zu bringen, anstatt sie dumpf in identifizierenden Begriffen verschwinden zu lassen. Legt man nämlich die Idee der Konstellation zugrunde, nach der die Gegenstände in ihrer Vermittlung begrifflich erfassbar sind, dann bedeutet das im Umkehrschluss auch, dass jeder Gegenstand eben dank dieser Vermittlung über sich hinaus auf das Ganze weist, dass durch ihn somit in seiner Wirklichkeit zu fassen ist. Das hat Adorno im Sinn, wenn er erklärt:

Inhaltlich als universale Vermittlung eines jeglichen Phänomens durch die gesellschaftliche Totalität, welche der Philosophie zur reinen Subjektivität sich verkehrt, steckt Allgemeines in jedem Besonderen. Jedoch die philosophische Erfahrung hat dies Allgemeine nicht, oder bloß abstrakt, und ist darum zum Ausgang vom Besonderen verhalten, ohne doch zu vergessen, was sie nicht hat, aber weiß. ${ }^{38}$

Wenn aber das Allgemeine in jedem Besonderen (und als nicht beschädigtes auch nur im Besonderen) aufzufinden ist, dann gilt das auch für die wahre, d.h. die der kritischen Reflexion entsprungene Subjektivität, die ihre Vermittlung in der Konstellation des Objektiven in ihr Verständnis von sich aufgenommen hat. Sie wird somit zum Zeichen eines partikularen Allgemeinen, im Stande, über sich hi-

37 Adorno: Vorlesung über Negative Dialektik, op. cit., S. 248.

38 Ibid., S. 252. 
naus zum Anderen zu zeigen. In Wahrheit schließen erst die moralischen Impulse einer solchen Allgemeinheit, so individuell sie dem naiven Blick auch erscheinen mögen, die Totalität mit ein.

Dr. Pierre Buhlmann ist assoziierter Wissenschaftler der Forschungsgruppe TRANSMIS/Erraphis (Universität Toulouse-Jean Jaurès). Er arbeitet zum Problem der Subjektivitätskonstitution in der ersten Generation der Frankfurter Schule und dem französischen Poststrukturalismus. 